\title{
Optical Absorption and Transmission Electron Microscopy Analysis of CdTe Quantum Dots Size Distribution
}

\author{
M. L. Redígolo, ${ }^{*}$ L. C. Barbosa, ${ }^{* *}$ A. M. de Paula, ${ }^{* * *}$ and C. H. Brito Cruz, \\ *IP\&D, Univap, Av. Shishima Hifumi, 2911, São José dos Campos-SP, Brazil, 12244-000. \\ ** GFURCO, IFGW, Unicamp, Campinas-SP, Brazil, 13083-970. \\ ${ }^{* * *}$ NanoLab, USF, Bragança Paulista-SP, Brazil, 12916-900.
}

Quantum confinement effects in semiconductor nanocrystals have attracted a significant amount of interest in the last few years, because of their new optical properties and also because of their potential for optical device applications. In this work the size-dependent optical properties of nanometer-scale semiconductor crystallites (or quantum dots) in doped glasses are investigated. The specimens studied are Cadmium Telluride (CdTe) quantum dots grown by processes of heat treatment [1].

The optical absorption of the samples were studied and the resulted spectra are compared with the calculated ones considering the transition energies from a spherical k•p model. Figure 1 shows the measured and calculated spectra. The inhomogeneous broadening due to the quantum dot size distribution and the homogeneous broadening due mainly to electron-phonon interactions have also been considered. The standard deviation for the size-distribution is 5,8 \% according to this analysis [2].

Transmission electron microscopy (TEM) is also used to directly measure the quantum dot size distribution. The TEM study was performed in the JEOL JEM-3010 microscope operating at $300 \mathrm{kV}$ at the Brazilian Synchrotron Light Laboratory. The TEM measurements were performed with the CdTe doped glass sample grinned to a very fine powder and mixed with alcohol. The suspension was dropped on a $\mathrm{NaCl}$ substrate, waited to dry and then have been coated with a $20 \AA$ carbon film. The substrate was removed by water and the left resulting thin film was collected and prepared for the TEM analysis.

The presence of the CdTe nanocrystals was first confirmed by energy dispersive X-ray spectroscopy (EDXS). In figure 2 (a), we can observe the EDS result presenting the Cadmium and the Tellurium lines. The TEM image of the CdTe nanocrystals is presented in figure 2 (b). The direct size distribution measurement (see figure 3) indicates a much larger standard size distribution than the value obtained from the optical absorption spectra. Some possibilities for this difference are proposed in this work such as effects of photodarkening for these samples and others [3].

\section{Reference}

[1] L. C. Barbosa et al., J. Non-Cryst. Solids 219 (1997) 205.

[2] M. L. Redígolo et al., Semicond. Sci. Tech. 14 (1999) 58.

[3] T. D. Krauss and L. E. Brus, Mat. Scien. Eng. B 69-70 (2000) 289. 


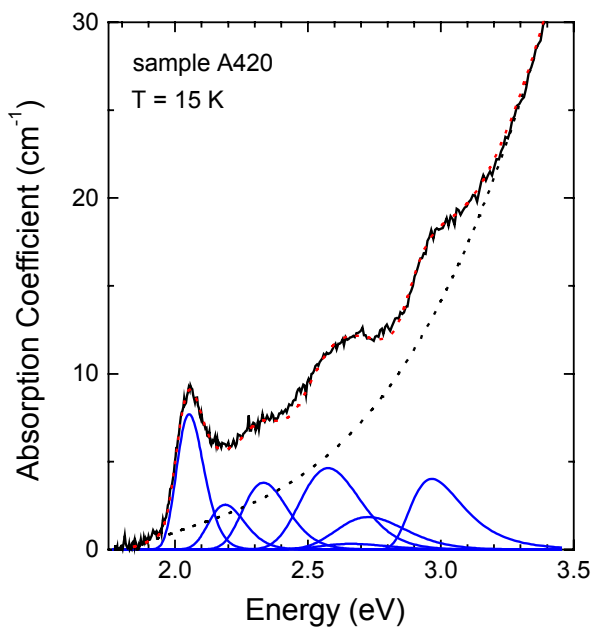

FIG. 1. Experimental and calculated optical absorption spectra for the CdTe quantum dots.

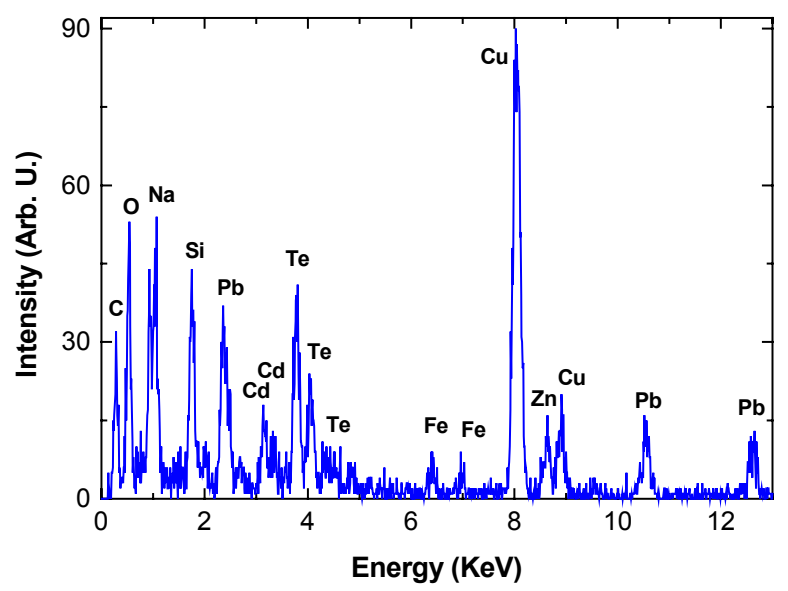

a)

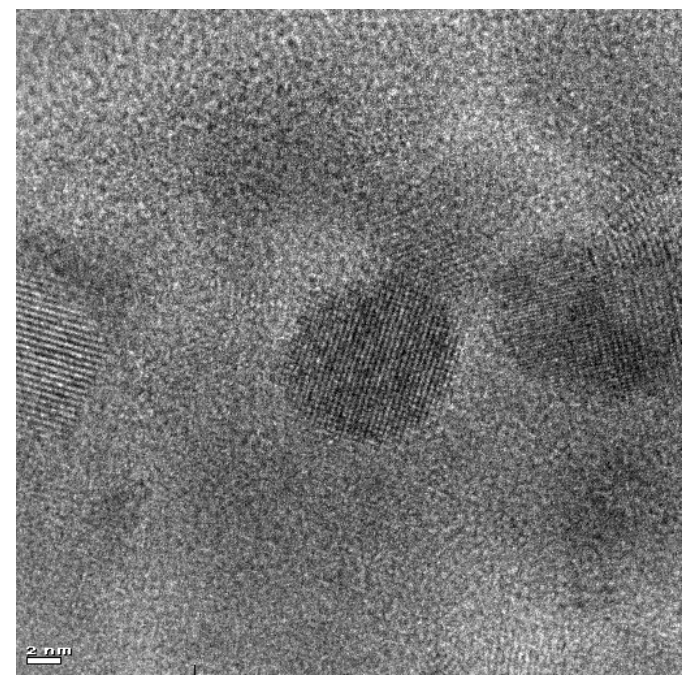

FIG. 2. (a) EDS analysis of the CdTe quantum dot samples; (b) TEM image of CdTe nanocrystals.

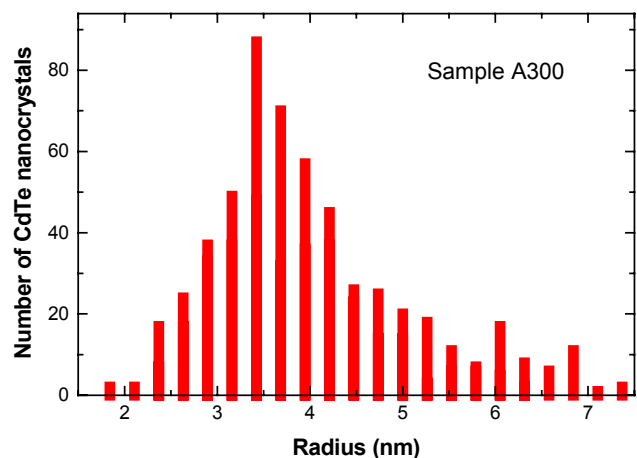

a)

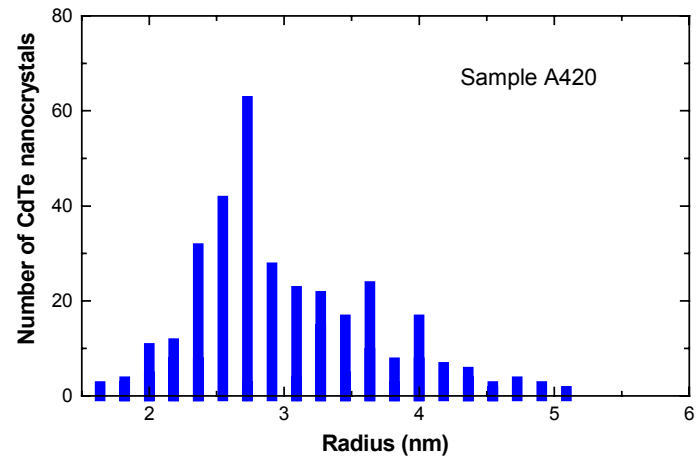

b)

FIG. 3. Size distribution of two CdTe quantum dot samples: (a) A300 and (b) A420. 